\title{
Modern molecular biology and respiratory bacterial infections: a revolution on the horizon
}

\author{
Timothy F Murphy
}

Respiratory bacterial infections are a major cause of worldwide morbidity and mortality in both developing and industrialised countries. Although antibiotics are often effective, this is not universally the case. Initial treatment is often empirical, being either of broad spectrum or targeted to an assumed pathogen. Ideally, the organism causing infection should be identified with accuracy as soon as possible, enabling specific treatment to be initiated.

Conventional microbiological techniques still fall short of this ideal on many occasions. For instance, acute lobar pneumonia in an adult with chronic obstructive lung disease with sputum containing predominantly Gram negative coccobacilli is likely to be diagnosed as Haemophilus influenzae infection. Microbiological confirmation and the results of sensitivity testing, however, may not be available for several days even if the organism grows in culture. In other patients with established lung diseases several organisms may be present and the results of microbiological culture may be influenced by the viability of the organism in vitro. Some may overgrow other pathogens, whereas more fastidious organisms, such as the anaerobes, may not survive in routine culture. Finally, some organisms, such as mycobacteria, may require several weeks in culture before they can be isolated and identified. Others, such as Legionella and Mycoplasma, may require cytological identification or retrospective diagnosis by detection of the immune response.

The development of molecular probes for specific DNA sequences of these organisms and the application of other molecular biological techniques offers an opportunity to obtain a more rapid and specific diagnosis in many bacterial infections. Furthermore, the techniques offer a unique opportunity to investigate the pathogenetic mechanisms in bacterial infection. With this knowledge we will be able to devise other therapeutic strategies as an alternative to antibiotic treatment.

The purpose of this article is to outline how advances in molecular biological techniques will change our understanding and management of respiratory bacterial infections. It will focus primarily on two areas: (1) the devel- opment of DNA probes for diagnosis; and (2) the application of mutagenesis studies to elucidate molecular mechanisms of microbial pathogenesis.

\section{DNA probes in the diagnosis of respiratory bacterial infections ROLE OF DNA PROBES IN DIAGNOSIS}

For the reasons I have outlined, the development of a probe that is species specific would have immediate application in the diagnosis of pneumonia. Indeed, diagnostic kits using DNA probes that detect Mycoplasma pneumoniae and Legionella pneumophila are now commercially available and are both sensitive and specific. ${ }^{1-4}$ The refinement of these probes and the development of similar probes to detect Chlamydia pneumoniae and mycobacteria are likely to follow and will have important and immediate application in the diagnosis of these infections. ${ }^{5}$

A similar problem, though not strictly bacterial, arises in the diagnosis of Pneumocystis carinii infection, which is difficult with conventional techniques. Recent studies have shown that the organism can be identified in tissue by in situ hybridisation as well as in secretions. ${ }^{67}$ The diagnosis of mycobacterial infection is often delayed because several weeks are required for in vitro cultivation. DNA probes for identifying mycobacteria will be a considerable improvement over current technology. Probes for distinguishing $M$ tuberculosis rapidly from the "atypical" mycobacteria will have an important effect on the management of patients, particularly in the setting of AIDS.

When numbers of the organism are low, the relevant sequences can be amplified by the polymerase chain reaction before hybridisation with the relevant probe. ${ }^{8}$ The sensitivity of this latter technique is such that a positive result can be obtained with even one organism. Whereas this may be an advantage when organisms are scanty, it may also prove a disadvantage if the organism is not replicating in the lung and causing the infection. Further study and refinement will be required to clarify the role of the polymerase chain reaction in the diagnosis of respiratory infection.

Unfortunately, most cases of pneumonia in adults are caused by bacteria that also colonise 
Potential advantages of analysis of lung secretions with DNA probes over conventional culture

1 Faster results.

2 Eliminates bacterial overgrowth as a problem.

3 Quantitates bacteria more readily.

4 In situ studies with tissue samples to confirm bacterial invasion.

5 Identify selected serotypes associated with disease.

the upper airway in the absence of disease. Consequently, the presence of the bacterium in a sputum sample does not necessarily establish the diagnosis. What, then, is the role for DNA probes in the diagnosis of bacterial pneumonia in these circumstances?

In most instances the time honoured method of Gram staining and culture of sputum samples is used to predict the aetiology of bacterial pneumonia in adults, though there are serious limitations to this approach. ${ }^{914}$ In the absence of invasive diagnostic techniques, however, studies of sputum are still all we have available and these are widely used by physicians to guide treatment. The application of modern molecular biological techniques to sputum studies offers several advantages over Gram staining and culture, particularly when fastitious or multiple organisms are present.

Molecular probes will allow clinicians to detect potential pathogens more reliably than Gram staining and more quickly than routine culture. In addition, these techniques have the potential to improve the quality of the information obtained from sputum by allowing bacteria to be quantified more readily in a whole sputum specimen, whereas Gram staining and culture sample only a portion of the specimen.

The DNA from the whole sputum sample can be extracted, and unique sequences of DNA corresponding to pathogens can be quantified in this aqueous extract. Where numbers of organisms are small, the amount of potential DNA can be amplified by the polymerase chain reaction before molecular probes are used as mentioned above. Analysis with DNA probes overcomes the problem of overgrowth of some bacterial species on culture plates, a major problem in identifying more fastidious bacteria such as the pneumococcus, particularly when swarming bacteria are present. When invasive techniques such as lung biopsy are used, in situ hybridisation with probes will allow pathogens to be identified in tissue, thereby confirming that they are invasive organisms. Finally, as we learn more about the pathogenesis of respiratory bacterial pathogens, certain serotypes of some bacteria may prove to be capable of causing pneumonia whereas other serotypes of the same species may be capable only of colonisation of the upper airway. Development of probes to identify such serotypes in secretions
Figure 1 Schematic diagram of the analysis of a sputum sample with DNA probes. Probes will detect DNA sequences that are unique to each of the bacterial species that cause respiratory tract infections.

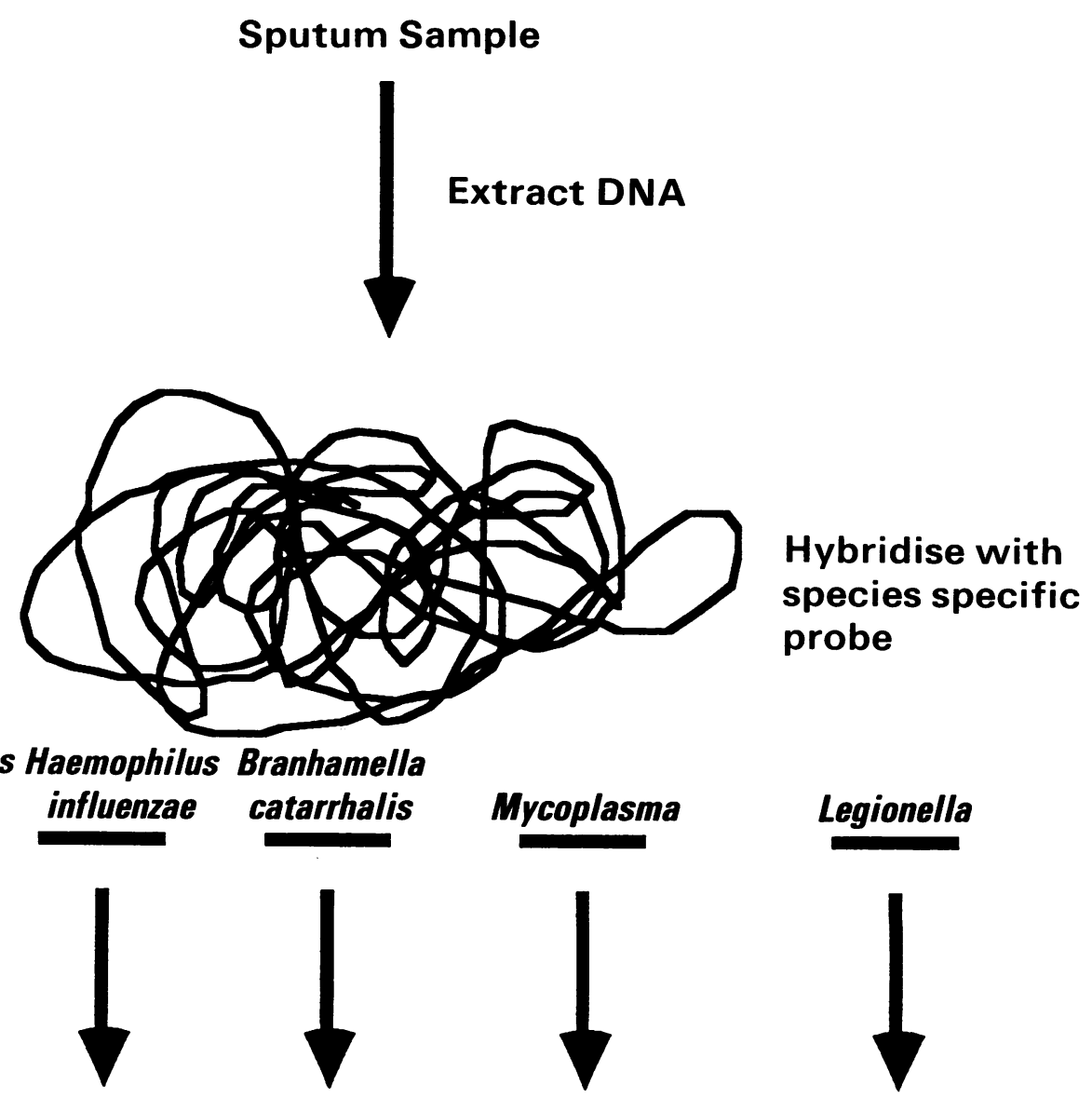

Positive result identifies bacterium 
Figure 2 Schematic diagram depicting an experiment to assess the potential of antigen $A$ as a virulence factor in an animal model. The wild type and mutant strains are isogenic, differing only in the gene that encodes antigen $A$.

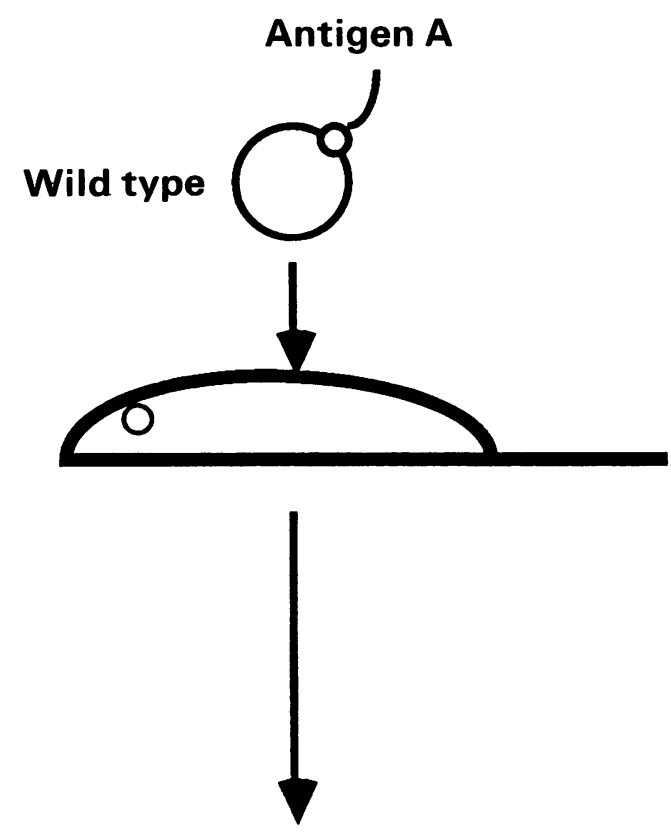

Disease

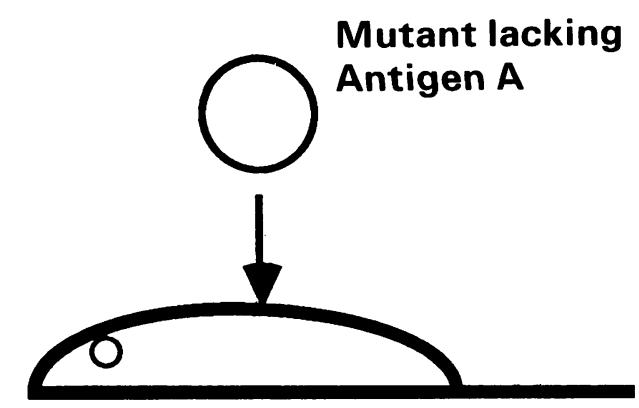

No disease

\section{Conclusion: Antigen $A$ is a virulence factor}

\section{Koch's Postulates}

Organism is present in association with the disease it causes

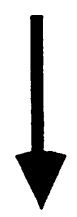

Causative agent is isolated and grown in pure culture

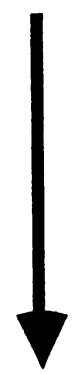

Inoculation with a pure culture reproduces disease in animal model

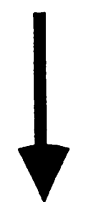

Same organism recovered from animal

\section{Molecular Koch's Postulates}

Trait under study is associated with pathogenic strains of the species

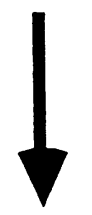

Specific inactivation (mutation) of the gene associated with the trait leads to measurable loss of virulence

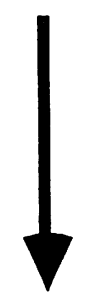

Reversal of gene mutation restores virulence 
Figure 4 Schematic diagram illustrating six methods for creating mutations in a fragment of cloned DNA. See text for explanation.

4(a) Oligonucleotide directed mutagenesis.
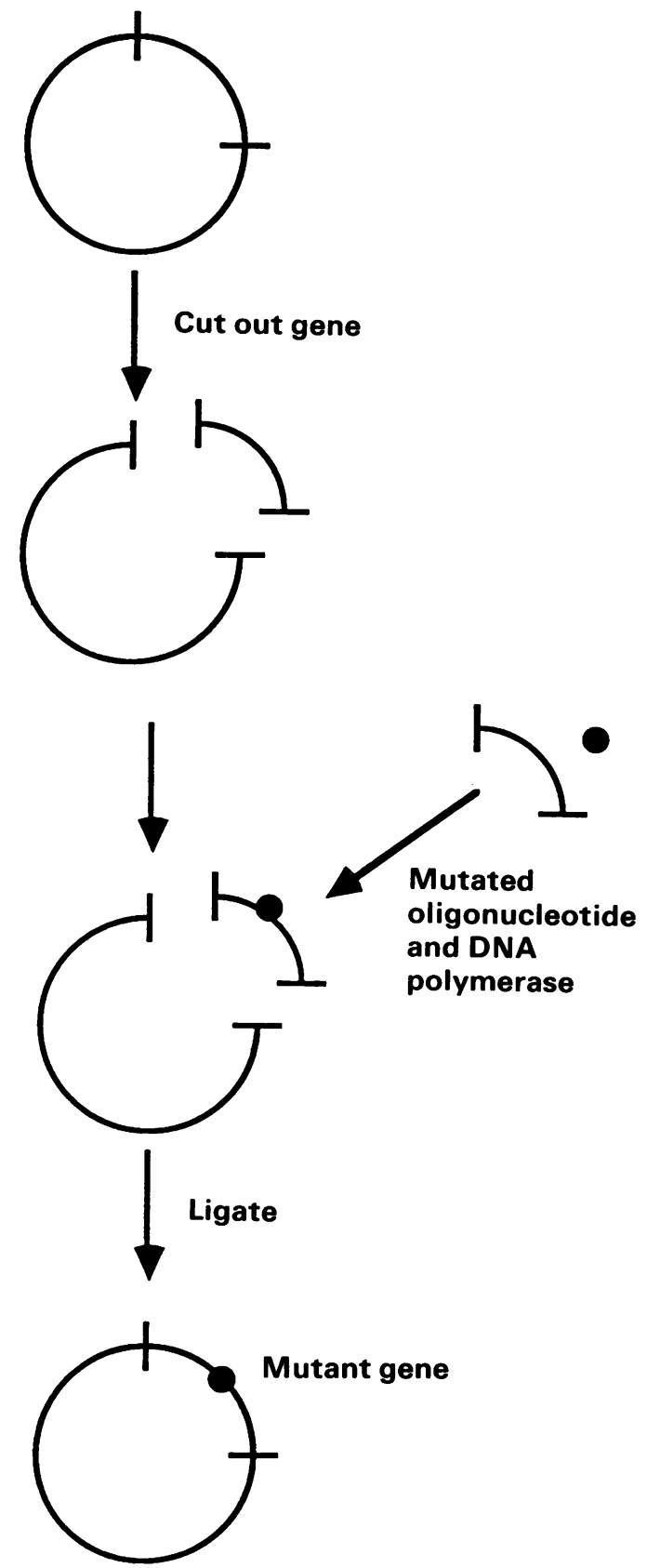

will improve our diagnostic capabilities and hence therapeutic interventions.

Thus the availability of DNA probes to identify bacterial respiratory pathogens in secretions and tissues can help to guide the clinician at the bedside in making decisions about antimicrobial treatment for patients with pneumonia. Some of the potential advantages of analysis of sputum samples with DNA probes rather than conventional culture are summarised in the table.

\section{DEVELOPMENT OF A DNA PROBE}

Developing a DNA probe to identify a bacterial species in a clinical specimen means identifying a DNA sequence that is unique for that species. This sequence must be present in all strains of the species being studied and must be absent in all other bacteria that might be present in the clinical sample. In addition, this sequence must be absent from the patient's tissues and secretions, such as mucus in sputum, so that false positive results are not obtained by hybridisation of the probe with host material. An example of this principle is provided by molecular studies of the P6 protein of Haemophilus influenzae. P6 is an outer membrane protein that is present on the surface of all strains of $H$ influenzae. ${ }^{15} 16$ P6 has attracted interest as a potential vaccine antigen because it appears to generate protective antibody. ${ }^{17-19}$ In the course of our studies on P6 we cloned and determined the DNA sequence of the gene encoding P6 from one strain of $H$ influenzae. ${ }^{20}$ Next we studied the extent to which the P6 genes from other strains of $H$ influenzae are similar to or different from each other. To determine the degree of molecular conservation of the gene among strains of $H$ influenzae, we performed restriction fragment length polymorphism analysis on 40 strains of diverse origin as follows.

Five oligonucleotides of 17 bases each were synthesised. These oligonucleotides corresponded to DNA sequences from five different parts of the P6 gene from the $N$ terminus of the protein to the $C$ terminus. The probes were radiolabelled and used to probe DNA from 40 different strains of $H$ influenzae in Southern blot assays to determine whether any parts of the P6 gene were similar among strains. The DNA from these strains had been previously digested with restriction enzymes to yield fragments of DNA of defined sizes on the basis of the known sequence of the P6 gene. Three of the five oligonucleotides hybridised with fragments of DNA of identical size in all 40 strains.

After identifying three probes that hybridise with DNA of all strains of $H$ influen$z a e$, we determined whether these probes were specific for $H$ influenzae. DNA was purified from various bacterial species and tested with the probes. They were highly specific for $H$ influenzae, showing no hybridisation with various other bacterial species. These probes also hybridise with $H$ influenzae DNA in whole bacterial colonies, making it unnecessary to purify DNA from strains to be tested. These probes therefore show promise as diagnostic reagents for $H$ influenzae in sputum. A slightly different approach has been used by Terpstra et al, ${ }^{21}{ }^{22}$ who used labelled DNA from the whole genome to probe sputum samples and effectively identified $H$ influenzae in sputum.

It will be straightforward to use these methods to identify sequences that are unique to each of the bacterial species that cause pneumonia. We can then envisage that a secretion sample submitted to the laboratory will be rapidly analysed with probes that will detect the presence of the major respiratory bacterial pathogens. This idea is depicted diagrammatically in figure 1 . As a result, the clinician will know the results of a sputum analysis with regard to the presence or absence of potential pathogens in a matter of hours, (the exact time depending on the indicators used to label the probes) rather than days as with conventional bacterial culturesor even weeks for organisms such as the mycobacteria. 

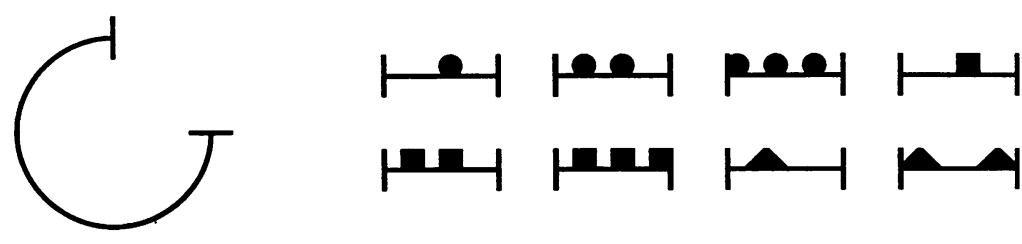

Cut out gene
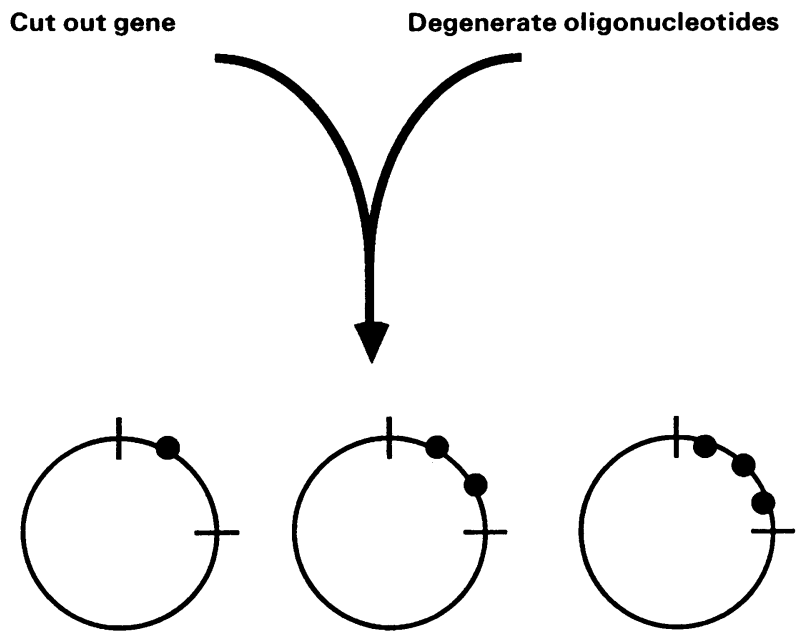

etc

Multiple mutations in a small region

4(b) Mutations with degenerate oligonucleotides.

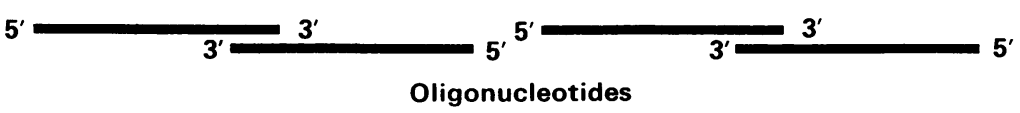

DNA polymerase :
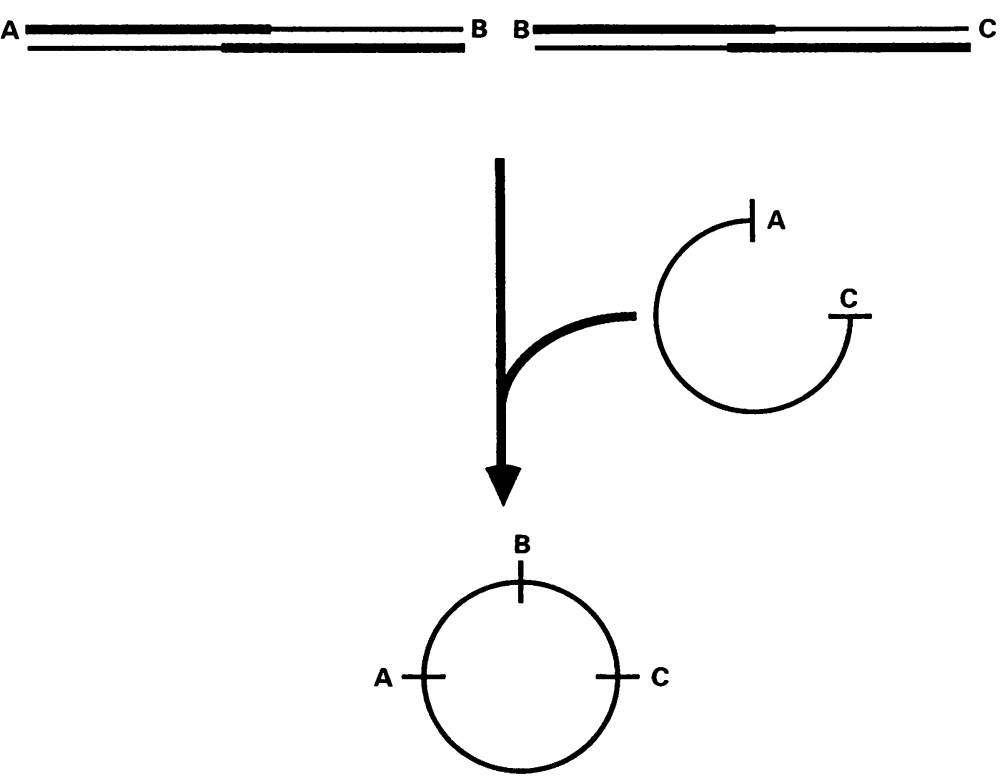

A through $\mathrm{C}$ is newly synthesised gene

4(c) Gene synthesis with long oligonucleotides.
Studies of mechanisms of microbial pathogenesis

Bacterial pathogens possess certain characteristics that give them the capacity to compete successfully with other bacteria, gain access to the host, avoid host defence mechanisms, and multiply within the host. Studies designed to identify and characterise the factors that allow respiratory bacterial pathogens to cause disease represent a critical initial step in understanding pathogenesis at the molecular level and will lead directly to the development of strategies of intervention in these infections in the form of new therapies and vaccines.

The application of modern molecular biological techniques to studies of bacteria is leading to new and important information in several ways. ${ }^{23}$ The capacity to clone the gene that encodes a microbial protein antigen, for example, can lead to a large amount of information about the function of that protein for the bacterium and the potential role of the molecule as a virulence factor. Determination of the DNA sequence of the gene allows us to learn the entire amino acid sequence of a protein antigen, and structure-function relationships can be elucidated. We can manipulate and investigate regulation of the expression of potential virulence factors. Studies of the regulation of expression of bacterial antigens are particularly important in that bacteria have an enormous capacity to express different antigens in different environmental conditions. Cloning of the gene encoding a protein allows us to identify the precise portions of the molecule (epitopes) that are recognised and bound by antibodies. Epitope mapping is important in studying bacterial antigens as targets of the human immune response to infection. Finally, the cloned gene of a putative virulence factor can be studied by inactivating or altering the gene. This is a powerful technique and will be considered in more detail.

In the classic studies designed to identify microbial virulence factors pathogenic and non-pathogenic bacterial strains have been compared with regard to their ability to cause disease. Identifying factors associated with virulence in this way is limited by the many differences among bacterial strains even of the same species. The molecular genetic approach allows the investigator to identify accurately the individual molecules concerned with pathogenesis. The most widely used strategy is to create a mutant that differs from the parent strain in a single molecule. The mutant and parent strains are then compared with regard to their ability to cause disease in an appropriate laboratory model. This concept is depicted diagrammatically in figure 2 .

Koch's postulates were originally formulated to establish a causal relation between an organism and a specific disease. The ability to manipulate individual genes and molecules to identify and study virulence factors has led to the concept of "molecular Koch's postulates". ${ }^{24}$ These are designed to be applied to virulence factors, to see whether they have a role in the pathogenesis of infection by an organism. Simply stated, molecular Koch's 

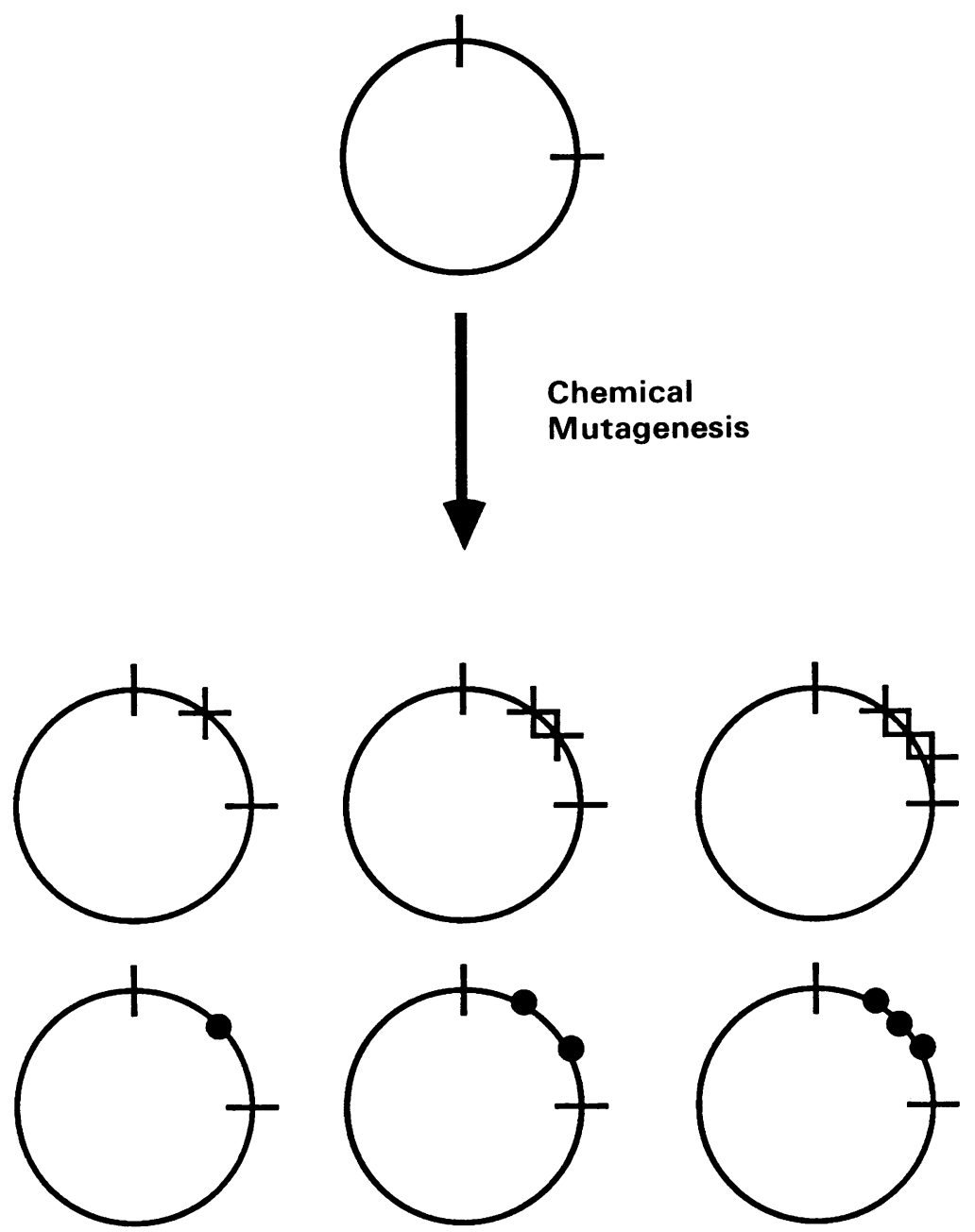

Multiple Mutations

4(d) Region specific chemical mutagenesis.

postulates are as follows: (1) The trait under study is associated with pathogenic strains of the species; (2) specific inactivation (mutation) of the gene associated with the trait leads to a measurable loss of virulence; (3) reversal of the gene mutation restores virulence. These postulates are depicted, along with the classic Koch's postulates, in figure 3.

MUTAGENESIS OF CLONED DNA

The power of molecular biological techniques to determine the mechanisms underlying microbial pathogenesis rests in the ability of investigators to manipulate the genes of putative virulence factors. Once the gene for a virulence factor has been identified, isolated, and cloned into a vector, such as a plasmid, the gene can be manipulated in various ways. A particularly useful approach is to alter or inactivate the cloned gene. The parent strain is then transformed with the altered gene by selecting for antibiotic resistance to create a mutant. The effect of loss of the virulence factor on the bacterium's ability to cause disease in an animal model is then assessed. This allows the investigator systematically to assess the potential role of individual molecules that mediate pathogenesis. Several strategies for altering or inactivating genes can be used. Some of these are summarised in figure 4 and detailed below. Other strategies exist, but this brief consideration of six methods provides a survey of the kinds of manipulations that can be performed.

Oligonucleotide directed mutagenesis (fig 4a) This method allows a DNA sequence to be altered in a defined way. An oligonucleotide containing the mutation of interest is synthesised and incorporated into the gene to be studied. This method is valuable for studying the effects of specific changes in a DNA molecule. It is also useful for introducing new restriction enzyme sites in defined points in the DNA molecule to facilitate future studies.

Mutagenesis with degenerate oligonucleotides (fig $4 b$ ) Synthetically derived mixtures of oligonucleotides are generated and used to create genes with random mutations within a defined region. The frequency of the mutations is determined by the conditions used for oligonucleotide synthesis. This method is useful for generating a large number of mutations within a small region of DNA. Creating mutants in several places within a region of DNA that codes for a single molecule allows one to identify the portion of the gene product that is involved in a particular pathogenic mechanism.

Gene synthesis with long oligonucleotides (fig 4c) This procedure makes it possible to "custom design" genes. Long oligonucleotides (up to 400 nucleotides) with homologous ends are synthesised and converted into double stranded DNA that is suitable for cloning. The cloned DNA can be studied in isolation or in expression systems, or be introduced into bacterial species.

Region specific chemical mutagenesis (fig 4d) Single stranded DNA containing the region of interest is treated with various chemicals that alter the DNA. When oligonucleotides are used as primers the altered region can be copied and then cloned. The mutation frequency depends on the duration and concentration of the chemical treatment. This method is useful for generating random mutations in a larger region ( $1-3$ kilobases) of DNA.

Nested sets of deletion mutants (fig $4 e$ ) In this method mutants that lack progressively more sequences from one end of the DNA molecule are generated. The DNA of interest is digested for varying time intervals with an exonuclease that cleaves nucleotides sequentially from the end of the molecule. The DNA molecules of varying length are then cloned. This approach is useful for identifying regulatory sequences and for identifying epitopes recognised by antibodies.

Linker scanning mutagenesis (fig $4 f$ ) In this method mutations are created by inserting short sequences containing new restriction 


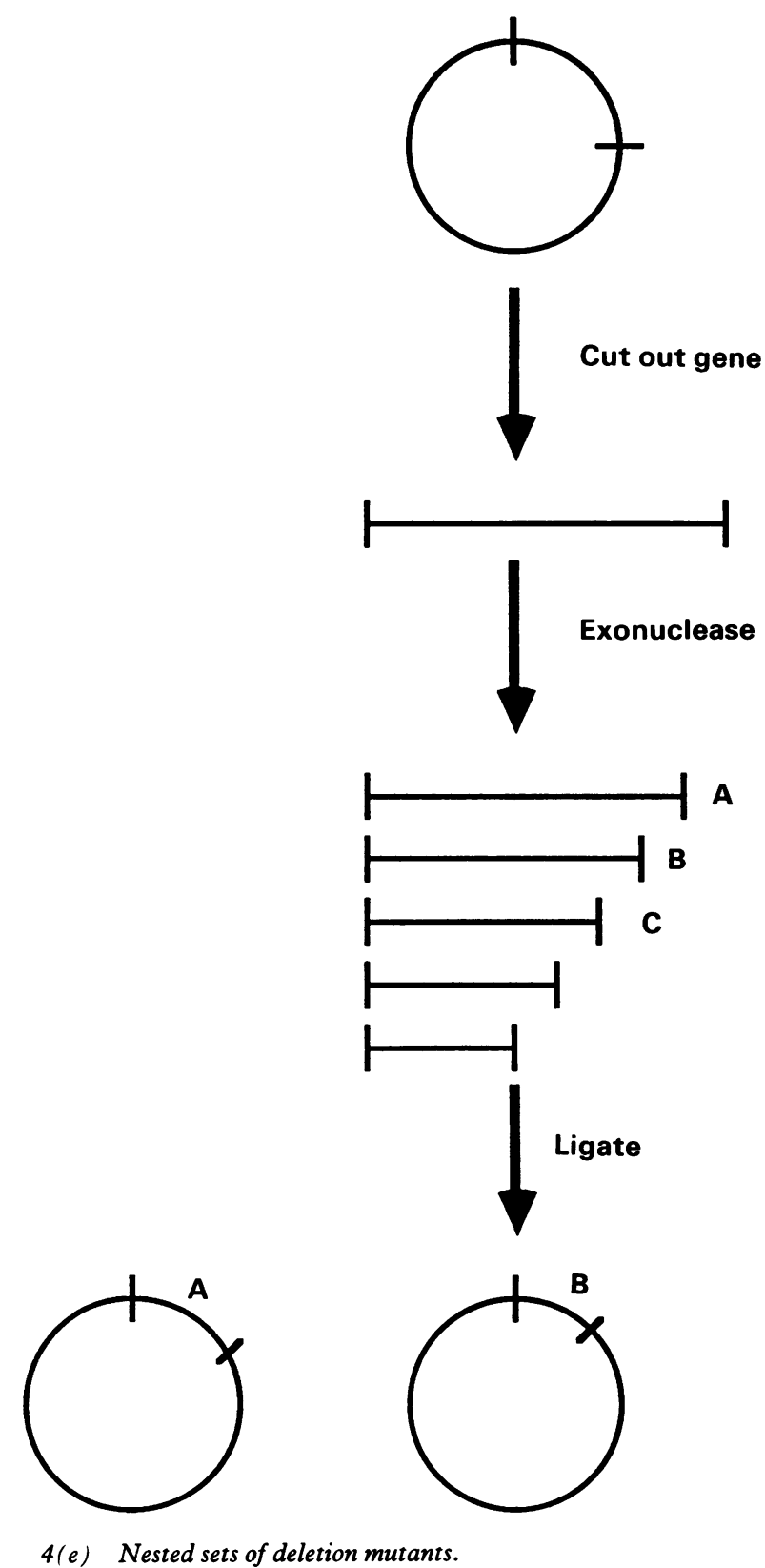

sites into defined regions of the cloned DNA molecule. Creating and studying a series of linker scanning mutations throughout a region also allows identification of functionally important sequences of a gene.

The application of these principles is illustrated in a series of experiments performed by McDaniel, et al with Streptococcus pneumoniae. $^{25-28}$ Preliminary studies had suggested that an antigen called pneumococcal surface protein A (PspA) was a virulence factor for the organism. To assess directly the role of PspA in the virulence of pneumococcal infection in the mouse model, mutant strains (in which the PspA gene was inactivated) were constructed. These mutants and the parent strain were isogenic, differing only in the PspA gene. The virulence of $\mathrm{PspA}^{-}$mutants was compared with that of their parent strains (WU2 and DBL5) in the mouse model of pneumococcal infection. None of the mice that received the $\mathrm{PspA}^{-}$ mutants died whereas all mice that received the corresponding parent strains died. ${ }^{25}$ The authors concluded that PspA is a major deter-
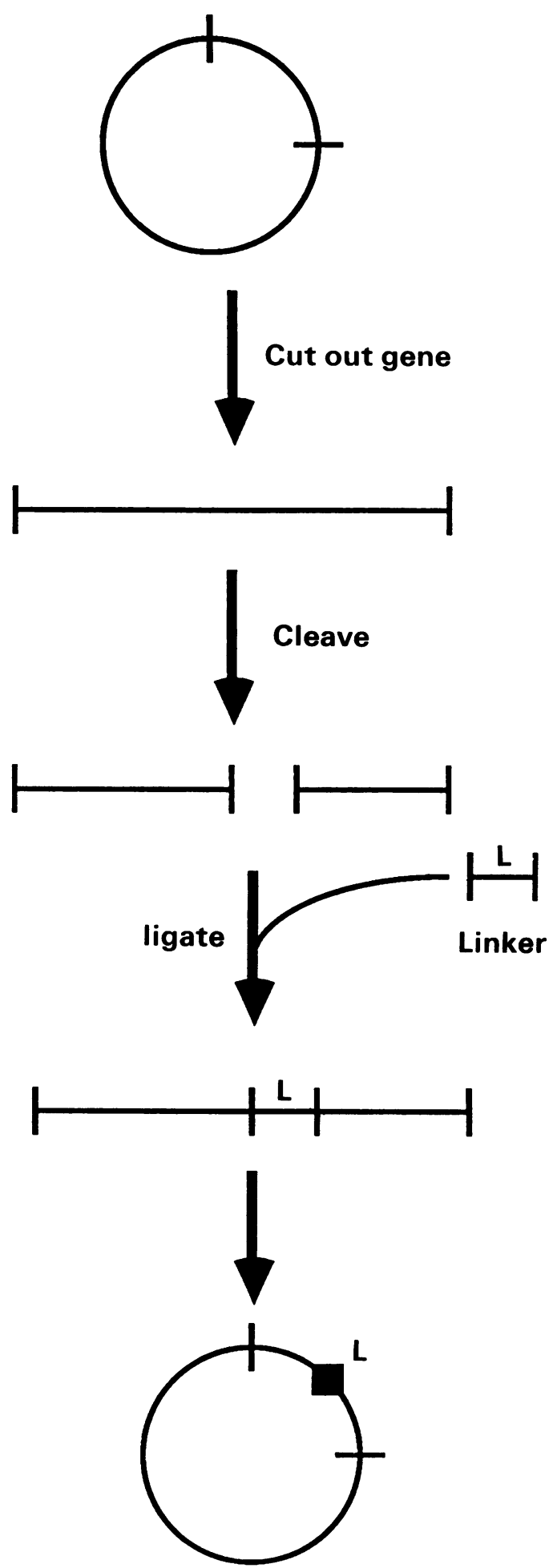

\section{Altered sequence with new restriction site}

4(f) Linker scanning mutagenesis.

minant of virulence for these pneumococcal strains. These experiments represent an elegant example of the use of molecular biological techniques to identify bacterial virulence factors.

Numerous other examples have been published in recent years. These include studies of the P6 protein in $H$ influenzae, ${ }^{15-20}$ the $\mathrm{P} 1$ adhesin of $M$ pneumoniae, ${ }^{29-31}$ polysaccharide microcapsules in Staphylococcus aureus, ${ }^{32} 33$ pili as adhesins in Gram negative bacilli, ${ }^{34-37}$ and 
many others. The major impact of these studies from a practical standpoint will be the identification of bacterial antigens that will induce protection from infection. These observations will guide vaccine development in an effort to prevent bacterial pneumonia in populations at risk.

Once the appropriate antigens for vaccines are developed, the design and production of the vaccines themselves will be facilitated by the application of recombinant DNA technology. ${ }^{38}$ Genes encoding vaccine antigens can be cloned and expressed in prokaryotic cells, eukaryotic cells (yeast), or mammalian cells. This approach has yet to be applied to bacterial antigens but it has been used for viruses. For instance, a widely used recombinant vaccine for hepatitis B is expressed in yeast. Alternatively, antigens can be inserted and delivered in viruses of low pathogenicity, such as vaccinia virus, resulting in a live recombinant vaccine that produces an immune response to the inserted antigen.

\section{Conclusions}

The recent extraordinary advances in molecular biological techniques is providing investigators with the tools to study molecular mechanisms of microbial pathogenesis. The application of these techniques to the study of respiratory bacterial pathogens will have a profound impact on these infections. This will be manifested primarily in the development of better methods for diagnosis and a more detailed understanding of mechanisms of pathogenesis, which will lead to vaccine development. In view of the importance of respiratory bacterial infections as a worldwide health problem, it is appropriate to continue to devote substantial resources to the problem.

1 Hyman HC, Yogev D, Razin S. DNA probes for detection and identification of Mycoplasma pneumoniae and Mycop-

2 Harris R, Marmion BP, Varkanis G, Kok T, Lunn B, Martin $\mathrm{J}$. Laboratory diagnosis of Mycoplasma pneumoniae infection. Epidemiol Infect 1988;101:685-94.

3 Dular R, Kajioka R, Kasatiya S. Comparsion of gen-probe commercial kit and culture technique in the diagnosis of Mycoplasma pneumoniae infection. J Clin Microbiol 1988, 26:1068-9.

4 Edelstein PH. Evaluation of the gen-probe DNA probe for the detection of legionellae in culture. J Clin Microbiol 1986;23:481-4.

5 LeBar W, Herschman B, Jemal C, Pierzchala J. Comparison of DNA probe, monoclonal antibody enzyme immunoassay, and cell culture for the detection of Chlamydia assay, and cell culture for the detection of

6 .Edman JC, Kovacs JA, Masur H, Santi DV, Elwood HJ, Sogin ML. Ribosomal RNA sequence shows Pneumocystis carinii to be a member of the fungi. Nature 1988:334: 519-22.

7 Wakefield AE, Hopkin JM, Burns J, Hipkiss JB, Stuart TJ, Moxon ER. Cloning of DNA from Pneumocystis carinii. J Infect Dis 1988;158:859-62.

8 Saiki RK, Gelfand DH, Stoffel S, et al. Primer-directed enzymatic amplification of DNA with thermostable DNA polymerase. Science 1988;239:487-91.

9 Bartlett JG. Diagnosis of bacterial infections of the lung. Clin Chest Med 1987;8:119-34.
10 Tobin MJ. Diagnosis of pneumonia: techniques and problems. Respir Infect 1987;8:513-27.

11 Balows A. A reassessment of sputum specimens for laboratory diagnosis of pneumococcal pneumonia. Eur $J$ Clin Microbiol 1986;5:275-6.

12 Lentino JR, Lucks DA. Nonvalue of sputum culture in the management of lower respiratory tract infections. $J$ Clin Microbiol 1987;25:758-62.

13 Barrett-Connor E. The nonvalue of sputum culture in the diagnosis of pneumococcal pneumonia. Am Rev Respir Dis diagnosis of pneum

14 Murphy TF, Fine BC. Bacteremic pneumococcal pneumonia in the elderly. Am J Med Sci 1984;288:114-8.

15 Murphy TF, Nelson MB, Dudas KC, Mylotte JM, Apicella MA. Identification of a specific epitope of Haemophilus influenzae on a 16,600 -dalton outer membrane protein. $J$ Infect Dis 1985;152:1300-7.

16 Murphy TF, Bartos LC, Campagnari AA, Nelson MB, Apicella MA. Antigenic characterization of the P6 protein of nontypable Haemophilus influenzae. Infect Immun 1986; 54:774-9.

17 Murphy TF, Bartos LC, Rice PA, Nelson MB, Dudas KC, Apicella MA. Identification of a 16,600-dalton outer membrane protein on nontypable Haemophilus influenzae as a target for human serum bactericidal antibody. J Clin Invest 1986;78:1020-7.

18 Munson RS Jr, Granoff DM. Purification and partial characterization of outer membrane proteins P5 and P6 from Haemophilus influenzae type b. Infect Immun 1985; 49:544-9.

19 Nelson MB, Murphy TF, van Keulen H, Rekosh D, Apicella MA. Studies on P6, an important outer-membrane protein antigen of Haemophilus influenzae. Rev Infect Dis 1988;10:S331-6.

20 Nelson MB, Apicella MA, Murphy TF, van Keulen H, Spotila LD, Rekosh D. Cloning and sequencing of Haemophilus influenzae outer membrane protein P6. Infect Immun 1988;56:128-34.

21 Terpstra WJ, Schoone GJ, Schegget JT, Van Nierop JC, Griffioen RW. In situ hybridization for the detection of Haemophilus in sputum of patients with cystic fibrosis. Scand J Infect Dis 1987;19:641-6.

22 Terpstra WJ, Groeneveld K, Eijk PP, et al. Comparison of two nonculture techniques for detection of Hemophilus two nonculture techniques for detection

23 Engleberg NC, Eisenstein BI. The impact of new cloning techniques on the diagnosis and treatment of infectious diseases. N Eng J Med 1984;311:893-901.

24 Falkow S. Molecular Koch's postulates applied to microbial pathogenicity. Rev Infect Dis 1988;10:S274-6.

25 Briles DE, Yother J, McDaniel LS. Role of pneumococcal surface protein $A$ in the virulence of Streptococcus pneumoniae. Rev Infect Dis 1988;10:S372-4.

26 McDaniel LS, Scott G, Widenhofer K, Carrol J, Briles DE. Analysis of a surface protein of Streptococcus pneumoniae recognized by protective monoclonal antibodies. Microbial Pathogen 1986;1:519-21.

27 McDaniel LS, Scott G, Kearney JF, Carrol J, Briles DE. Monoclonal antibodies against protease-sensitive pneumococcal antigens can protect mice from fatal infecpneumococcal antigens can protect mice from fatal infec160:386-97.

28 McDaniel LS, Yother J, Vijayakumar M, McGarry L, Guild WR, Briles DE. Use of insertional inactivation to facilitate
studies of biological properties of pneumococcal surface studies of biological properties of pneumococcal

29 Hu PC, Cole RM, Huang YS, et al. Mycoplasma pneumoniae infection: role of a surface protein in the attachment of organelle. Science 1982;216:313-4.

30 Trevino LB, Haldenwang WG, Baseman JB. Expression of Mycoplasma pneumoniae antigens in Escherichia coli. Infect Immun 1986;53:129-34.

31 Su CJ, Tryon VV, Baseman JB. Cloning and sequence analysis of cytadhesin P1 gene from Mycoplasma pneumoniae. Infect Immun 1987;55:3023-9.

32 Lee JC, Betley MJ, Hopkins CA, Perez NE, Pier GB. Virulence studies, in mice, of transposon-induced mutants of Staphylococcus aureus differing in capsule size. mutants of Staphylococcus aureus

33 Lee JC, Perez NE, Hopkins CA, Pier GB. Purified capsular polysaccharide-induced immunity to Staphylococcus aureus infection. J Infect Dis 1988;157:723-30.

34 Sastry PA, Pearlstone JR, Smillie LB, Paranchych W. Amino acid sequence of pilin isolated from Pseudomonas aeruginosa PAK. FEBS Lett 1983;151:253-6.

35 Woods DE, Straus DC, Johanson WG, Berry VK, Bass JA. Role of pili in adherence of Pseudomonas aeruginosa to mammalian buccal epithelial cells. Infect Immun 1980; 29:1146-51.

36 Lindberg F, Lund B, Johansson B, Normak S. Localization of the receptor binding protein adhesin at the tip of the bacterial pilus. Nature 1987;328:84-7.

37 Hanson MS, Brinton CC Jr. Identification and characterization of $E$. coli type 1 pilus tip adhesin protein. Nature tion of $E$. coli typ

38 Fields BN, Chanock RM. What biotechnology has to offer vaccine development. Rev Infect Dis 1989;11:S519-23. 